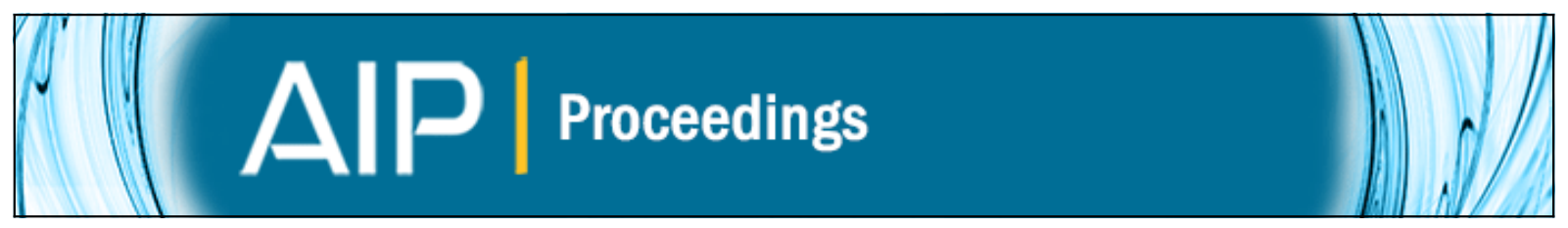

\title{
Sporicidal effects of iodine-oxide thermite reaction products
}

Rod Russell, Stephan Bless, Alexandra Blinkova, and Tiffany Chen

Citation: AIP Conference Proceedings 1426, 157 (2012); doi: 10.1063/1.3686244

View online: http://dx.doi.org/10.1063/1.3686244

View Table of Contents: http://scitation.aip.org/content/aip/proceeding/aipcp/1426?ver=pdfcov

Published by the AIP Publishing

Articles you may be interested in

Fluid dynamic modeling of nano-thermite reactions

J. Appl. Phys. 115, 104903 (2014); 10.1063/1.4867936

Modeling and simulation of pressure waves generated by nano-thermite reactions

J. Appl. Phys. 112, 094319 (2012); 10.1063/1.4764319

Transient ion ejection during nanocomposite thermite reactions

J. Appl. Phys. 106, 083306 (2009); 10.1063/1.3225907

Iron Oxide/Aluminum Fast Thermite Reaction

AIP Conf. Proc. 706, 871 (2004); 10.1063/1.1780375

Reaction Products of $y$ Picoline and lodine

J. Chem. Phys. 26, 331 (1957); 10.1063/1.1743294 


\title{
SPORICIDAL EFFECTS OF IODINE-OXIDE THERMITE REACTION PRODUCTS
}

\author{
R. Russell ${ }^{1}$, S. Bless ${ }^{1}$, A. Blinkova ${ }^{2}$, and T. Chen ${ }^{1}$ \\ ${ }^{1}$ Institute for Advanced Technology, The University of Texas at Austin, TX 78759 \\ ${ }^{2}$ Molecular Genetics \& Microbiology, The University of Texas at Austin, TX 78712
}

\begin{abstract}
Iodine pentoxide-aluminum thermite reactions have been driven by impacts at $1000 \mathrm{~m} / \mathrm{s}$ on steel plates $3 \mathrm{~mm}$ or thicker. This reaction releases iodine gas that is known to be a sporicide. To test the impact reactions for sporicidal effects, reactions took place in closed chambers containing dried Bacillus subtilis spores. The reduction in colony-forming units was dependent on the exposure time; long exposure times resulted in a $10^{5}$ decrease in germination rate. This was shown to be due to the gas exposure rather than the heat or turbulence. Sporicidal effectiveness was increased by adding neodymium and saran resin.
\end{abstract}

Keywords: iodine pentoxide, spores

PACS: 45.40.Gj, 82.40.Bj, 92.30.Wx.

\section{INTRODUCTION}

It is well established that some thermitic reactions can be driven by shock and impact loading. Therefore, it is plausible that iodineforming reactions of metals plus iodine pentoxide may also be shock driveable. If so, iodine reactions might have an application to the neutralization of biological-weapon agents, in particular anthrax spores. Investigation of this possible application was the motive for the work reported here.

\section{EXPERIMENTS}

The reactive materials (RM) of interest in this study were

$$
\begin{aligned}
& 10 \mathrm{Al}+3 \mathrm{I}_{2} \mathrm{O}_{5} \rightleftharpoons 5 \mathrm{Al}_{2} \mathrm{O}_{3}+3 \mathrm{I}_{2} \text { and } \\
& 10 \mathrm{Nd}+3 \mathrm{I}_{2} \mathrm{O}_{5} \rightleftharpoons 3 \mathrm{I}_{2}+5 \mathrm{Nd}_{2} \mathrm{O}_{3}
\end{aligned}
$$

The aluminum powder used was $99.5 \%$ pure 325 mesh supplied by Alfa Aesar. The iodine pentoxide was $99.99 \%$ pure purchased from Sigma-Aldridge. The neodymium was 99\% 325-mesh powder purchased from American Elements. All powder morphologies were examined with a scanning electron microscope (SEM). The $\mathrm{I}_{2} \mathrm{O}_{5}$ powder was comprised of 1-2 micrometer diameter amorphous grains of material that were agglomerated into particles that were tens of microns in dimension. The neodymium was comprised of micron-size crystallites.

Initial experiments to characterize thermal ignition of these materials are described in [1]. In [1] we also showed that these reactions can be initiated by impact onto thin $(1.5 \mathrm{~mm})$ steel plates at velocities exceeding $1 \mathrm{~km} / \mathrm{s}$.

The goal of the experiments was to evaluate the vulnerability of exposed, dried spores to the products of impact-driven reactions. A technique was therefore required to deposit spores into a 
container, expose the spores to reaction products, recover the spores, and assess their viability.

Our laboratory was operated in a biological safety level (BSL) 1 environment for all these experiments. Therefore, instead of using the extremely hazardous and infectious Bacillus anthracis, Bacillus subtilis was the bacterial strain tested. Bacillus subtilis spores suspended in sterile water were obtained from SGM Biotech, Inc., with a stated concentration of $10^{9} / \mathrm{mL}$. To determine the titer of viable spores in the stock suspension, serial dilutions were made in sterile water of 50 and 100 $\mu \mathrm{L}$. One-milliliter aliquots of the stock were stored at $4{ }^{\circ} \mathrm{C}$ with no loss of viability over 14 months.

Several types of tubes for the spore deposits were evaluated. Scintillation tubes were finally selected because of their wide mouth, convenient size, $16 \mathrm{~mm}$ diameter by $29 \mathrm{~mm}$ depth, and flat bottom. To isolate exposure variables of heat and gaseous products generated and to minimize solid products deposited into the scintillation tubes, screened caps were used for some of the tubes. Caps contained holes of $12.25 \mathrm{~mm}$ diameter with a copper mesh screen (Figure 1).

Test preparation involved the deposition of a layer of dry spores on the bottom of the tubes prior to exposure. This was done by starting from the $10 \% / \mathrm{mL}$ dilution stock and then transferring $50 \mu \mathrm{L}$ of this into the scintillation tubes. The theoretical number of spores in the tube was $5 \times 10^{7}$. The tubes were then dried overnight at $38^{\circ} \mathrm{C}$. Given the size of the drop, the deposition of spores on the glass was approximately one layer thick.

Due to the hydrophobic nature of the spores, the majority of them were expected to adhere to the bottom and sides of the scintillation tubes, which were used to hold the spores in the experiment chamber. For spore recovery, tubes were rehydrated with $2 \mathrm{~mL}$ of water and then agitated to suspend the spores in the water. The water could then be extracted for examination of the spore conditions. Standard plating procedures were used for transferring the rehydrated spores to agar plates. The plates were incubated overnight at $38^{\circ} \mathrm{C}$, and then counts were made of the colony forming units (CFU).

An issue that arose in analysis was the proper way to average data among different platings made from the Eppendorf tubes used for serial dilutions. In many experiments, there were CFUs in plates of quite different dilutions. For example, we might find $10 \mathrm{CFU}$ in a plate made from a $1,000 \times$ dilution and $1 \mathrm{CFU}$ in a plate from a $10,000 \times$ dilution. Clearly the plate with $10 \mathrm{CFU}$ is more statistically significant.

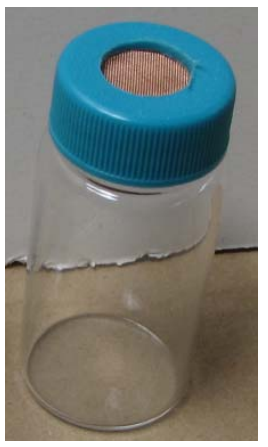

Figure 1. Scintillation tubes with screened caps were used to minimize the amount of product deposited into the tubes.

The approach was to consider each spore as a Bernoulli trial that may or may not produce a CFU. The probability of germination is low, so the success rate should have a Poisson distribution. Thus, the way the data was treated was to calculate the probability the given spore germinates $P$. It was computed from the total number of CFU observed divided by the total number of spores sampled. The formula for this is

$$
P=\frac{\sum n_{\mathrm{i}}}{\sum \frac{\delta N v_{\mathrm{i}} \Delta v_{\mathrm{i}}}{V_{0}}},
$$

where $N$ is the total number of spores in the test, $v_{\mathrm{i}}$ is the volume of the $\mathrm{i}^{\text {th }}$ Eppendorf tube, $\Delta v_{\mathrm{i}}$ is the volume removed with a pipette from that tube, $V_{0}$ is the rehydration volume, $n_{\mathrm{i}}$ is the number of CFU, and $\delta_{i}$ is the dilution.

In each experiment at least one control tube was set aside. The value for $P$ was computed from several different dilutions of these control tubes. From all the control tubes, the average value of $P$ was 1.03 with a standard deviation of 0.3 . These results validate that the rehydration technique does indeed recover the spores that may be adhered to the walls of the scintillation tubes. This result also 
justifies using the above formula to compute $P$ in spores that were exposed to reaction products.

\section{EXPERIMENTAL RESULTS}

The RMs were packed into brass cylinders for launch from a smooth-bore .50 caliber $(12.5 \mathrm{~mm}$ diameter) powder gun. The depth of the chamber in the brass cylinders was $29.9 \mathrm{~mm}$, and its volume was $2.4 \mathrm{~cm}^{3}$. The wall thickness was $1.3 \mathrm{~mm}$. A $0.65 \mathrm{~mm}$ thick lid was crimped in place. The RM was packed by careful hand tamping. The total package mass was approximately $20 \mathrm{~g}$. The projectile was filled with a stoichiometric mixture of the reactants, pressed to about $53 \%$ theoretical maximum density. The launch velocity of this projectile was measured with time-of-arrival screens placed about $2 \mathrm{~m}$ apart. In all tests, velocity was measured between 1,000 and $1,025 \mathrm{~m} / \mathrm{s}$.

Figure 2 is a to-scale sketch of the impact geometry; the set up was constructed in the impact laboratory at The University of Texas at Austin (www.iat.utexas.edu). The target tank was a 1.5 $\mathrm{mm}$ wall stainless steel drum. It contained a steel frame onto which the scintillation tubes were attached. The tubes were protected on the sides by wood blocks (because otherwise it was observed that most of them broke). In some experiments insulation was added to the target tank. In others there was a gap between the muzzle and the target tank so that the tank could vent to the laboratory. These modifications made little change in the recorded temperature history.

Figure 3 shows the temperature history of the midpoint of the outside wall for reactants (1). Measurements with other reactants did not change the peak temperature by more than 10 degrees, with similar decay times. Measurements of pressure in a vented chamber showed that the pressure decayed in about $0.25 \mathrm{~s}$. In a test experiment using a sealed chamber with an inert fill in the projectile, the temperature rise was only $40^{\circ} \mathrm{C}$; thus, about half of the thermal energy delivered to the chamber is due to the RM and the other half due to the gunpowder. Temperatures of this magnitude and duration should have no effect on spore viability [2].

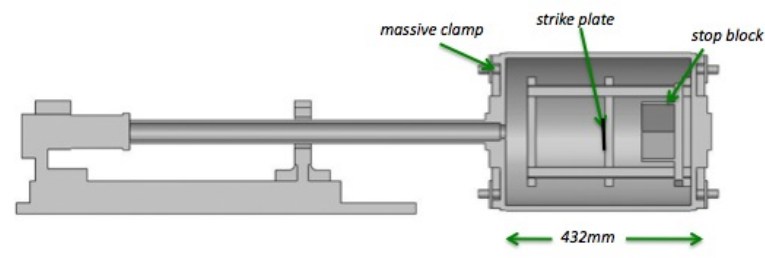

Figure 2. Experiment set up.

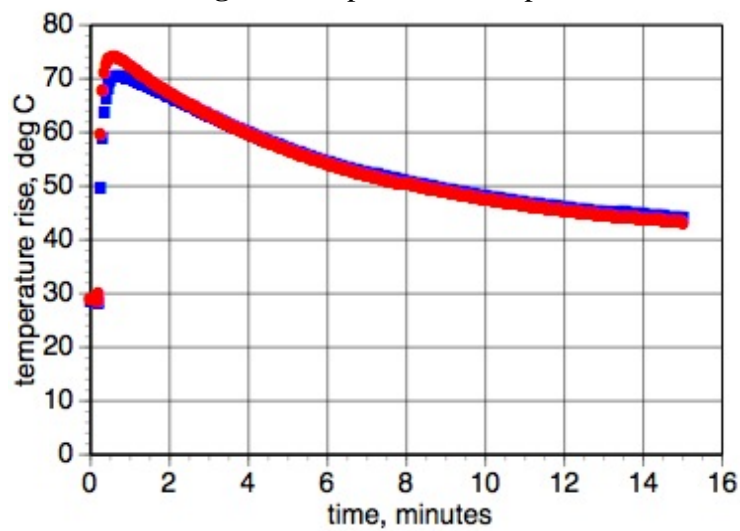

Figure 3. Wall temperature history when reactants (1) are fired into sealed chamber.

Initially, we considered buffering the water used for spore recovery to preclude damage to the spores from iodic acid. However, in the impact tests with iodine pentoxide and aluminum powder, the $\mathrm{pHs}$ of the rehydrated spore-containing tubes after the experiment were between the range of 3.5 and 5.5. This indicated that there was very minimal unreacted iodine pentoxide residue, since iodine pentoxide becomes iodic acid when mixed with water. We prepared some plates in which the reactants were added to agar. Iodine pentoxide was observed to prevent spore germination but aluminum did not. The recovered scintillation tubes from impact experiments often contained fine powder residue and often $0.5 \mathrm{~mm}$ size particles. In one test, spore-containing suspensions were introduced into these tubes after they were removed from the test chamber. These tubes were set aside for 24 hours and then serial dilutions were performed. The CFU counts were within scatter of total spores expected. Thus, spores were not killed by cooled or aged solid reaction products.

A series of impact tests was conducted to evaluate the sporicidal effects of the iodineproducing reactions. The time between the shots and the rehydration of the spores in the scintillation 
tubes was approximately one hour in all tests. The RMs tested were stochoimetric iodine oxide plus aluminum, stochoimetric bismuth oxide plus aluminum (which was used as an example of a reaction that produces hot turbulent gas that is not a potential sporicide), and a mixture consisting of $62 \%$ iodine pentoxide and aluminum, 30\% $\mathrm{I}_{2} \mathrm{O}_{5}$ and neodymium, and 8\% saran resin. The text tank was either vented or sealed for one hour before removal of the scintillation tubes. The results of these experiments in terms of spore germination are shown in Figure 4. The saran-containing mixture was effective at almost completely eliminating spore germination of the rehydrated spores.

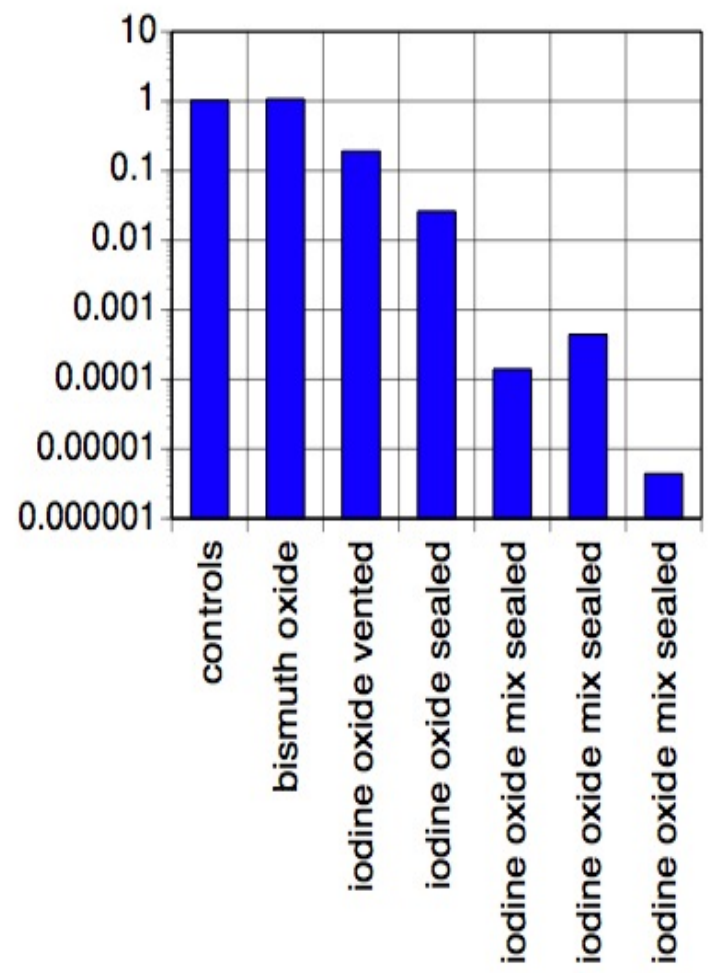

Figure 4. Ratio of CFU to theoretical number of spores present (from equation 3) for the tested chemicals. (Each test was a stoichiometric mixture with aluminum. See text above for “mix" definition.).

\section{CONCLUSIONS}

It appears clear that significant inhibition of germination occurs in the closed-range experiments with the enhanced iodine pentoxide thermite. Moreover, from the controls, the various control experiments make it clear that the reduction in CFU is not due to recovery techniques, turbulence, heat alone, or exposure to unreacted reactants but is instead due to the sporicidal action of the iodine gas.

\section{ACKNOWLEDGMENTS}

The research reported in this document was performed in connection with grant number HDTRA1-08-1-0013 with the Defense Threat Reduction Agency (DTRA). Any opinions, findings, and conclusions or recommendations expressed in this material are those of the authors and do not necessarily reflect the views of DTRA.

\section{REFERENCES}

1. Russell, R., Bless, S., and Pantoya, M., "ImpactDriven Thermite Reactions with Iodine Pentoxide and Silver Oxide,” Journal of Energetic Materials, vol. 29, no. 2, pp. 175-192, April-June 2011.

2. Alexander, C. A., Ogden, J. S., LeVere, M. A., Dye, C. F., and Kohler, D. F. "Thermal inactivation of aerosolized bacteria." Defense Special Weapons Agency. Columbus: Battelle, 1998. 